\section{Assessing the blood pressure waveform of the carotid artery using an ultrasound image processing method}

\author{
Effat Soleimani ${ }^{1}$, Manijhe Mokhtari-Dizaji ${ }^{1}$, Nasser Fatouraee ${ }^{2}$, Hazhir Saberi ${ }^{3}$ \\ 'Department of Medical Physics, Tarbiat Modares University, Tehran; 'Department of Medical \\ Engineering, Amirkabir University of Technology, Tehran; ${ }^{3}$ Department of Radiology, Imaging \\ Center of Imam Khomaini Hospital, Tehran Medical Sciences University, Tehran, Iran
}

Purpose: The aim of this study was to introduce and implement a noninvasive method to derive the carotid artery pressure waveform directly by processing diagnostic sonograms of the carotid artery.

Methods: Ultrasound image sequences of 20 healthy male subjects (age, $36 \pm 9$ years) were recorded during three cardiac cycles. The internal diameter and blood velocity waveforms were extracted from consecutive sonograms over the cardiac cycles by using custom analysis programs written in MATLAB. Finally, the application of a mathematical equation resulted in time changes of the arterial pressure. The resulting pressures were calibrated using the mean and the diastolic pressure of the radial artery.

Results: A good correlation was found between the mean carotid blood pressure obtained from the ultrasound image processing and the mean radial blood pressure obtained using a standard digital sphygmomanometer $(R=0.91)$. The mean absolute difference between the carotid calibrated pulse pressures and those measured clinically was $-1.333 \pm 6.548 \mathrm{~mm} \mathrm{Hg}$.

Conclusion: The results of this study suggest that consecutive sonograms of the carotid artery can be used for estimating a blood pressure waveform. We believe that our results promote a noninvasive technique for clinical applications that overcomes the reproducibility problems of common carotid artery tonometry with technical and anatomical causes.

Keywords: Ultrasonography; Carotid arteries; Arterial pressure; Computer assisted image processing; Calibration

\section{Introduction}

Blood pressure has been shown to be a strong indicator of cardiovascular health, particularly the coronary outcome. Increased systolic blood pressure (SBP) reflects changes in the arterial wall stiffness and vascular structure, whereas elevated pulse pressure (PP) predicts parallels between aortic atherosclerosis and the stiffening of conduit vessels [1]. Notwithstanding, the role of central pressure in clinical outcomes is increasingly recognized and central pressure seems to be more relevant to cardiovascular events than peripheral pressure [2]. Although invasive pressure waveform recordings

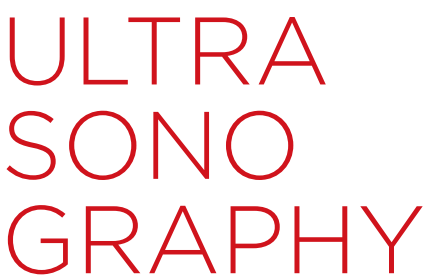

ORIGINAL ARTICLE

https://doi.org/10.14366/usg. 16019 pISSN: 2288-5919 - elSSN: 2288-5943 Ultrasonography 2017;36:144-152

Received: March 28, 2016

Revised: September 19, 2016

Accepted: September 20, 2016

Correspondence to:

Manijhe Mokhtari-Dizaji, PhD, Department of Medical Physics, Tarbiat Modares University, Jalal AleAhmad Highway, Nasr Bridge, Tehran 14115-11, Iran

Tel. $+98-21-82883893$

Fax. +98-21-88006544

E-mail: mokhtarm@modares.ac.ir

This is an Open Access article distributed under the terms of the Creative Commons Attribution NonCommercial License (http://creativecommons.org/ licenses/by-nc/3.0//) which permits unrestricted noncommercial use, distribution, and reproduction in any medium, provided the original work is properly cited.

Copyright @ 2017 Korean Society of Ultrasound in Medicine (KSUM)

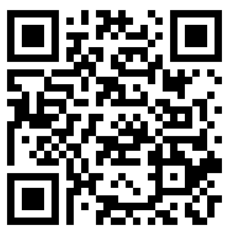

How to cite this article:

Soleimani E, Mokhtari-Dizaji M, Fatouraee N, Saberi H. Assessing the blood pressure waveform of the carotid artery using an ultrasound image processing method. Ultrasonography. 2017 Apr;36(2):144-152. 
are often considered the gold standard for central blood pressure (BP) measurements, invasive examinations have only been performed in a limited number of studies and not as part of daily clinical measurements [3]. Therefore, the pressure waveforms of the central arteries must be extracted from an analysis of surrogate waveforms for noninvasive pressure measurements. These waveforms are usually acquired using applanation tonometry of other easily available sites, particularly the radial artery, to which an arterial transfer function is applied $[4,5]$. Since the use of a universal transfer function (algorithm approved by Food and Drug Administration) is limited to the upper limbs, only the carotid artery and ascending aorta PP can be assessed via this technique [6].

Applanation tonometry is a well-known method to noninvasively record both central and peripheral pressure waveforms, which are then subjected to calibration or mathematical calculation. It is worth noting that the assessment of arterial pressure waveforms using applanation tonometry of the radial or carotid arteries can be technically and anatomically challenging and its measurement still lacks accuracy and has not found wide clinical application. This technique requires the operator to apply a hold-down mild pressure flattening the arterial wall to avoid the PP cushioning and obtain the optimal applanation, which occurs when the resulting waveform has a stable baseline $[3,4]$. Therefore, applanation tonometry has been reported to need a skilled investigator. Moreover, it requires specialized equipment and cannot be applied to all subjects and all arterial sites [7]. Furthermore, it assumes that the artery is compressible, which may not be true for carotid and conduit arteries [8].

However, other modern techniques and methods including echo tracking [9] and tissue Doppler imaging (TDI) [10] have made it possible to assess the central pressure waveform noninvasively. Today, high-resolution B-mode ultrasonic imaging of the carotid artery is familiar, feasible, and widely used in the diagnosis of atherosclerosis in imaging laboratories as it provides quantitative information of the intima-media thickness and lumen diameter and an assessment of the degree of stenosis as well as of plaque morphology [11]. Moreover, numerous studies have confirmed that temporal sequences of B-mode ultrasonic images analyzed with appropriate image processing techniques, may be used for extracting an arterial distention waveform as well as the arterial wall texture and elasticity features [12-14].

If arterial distention curves obtained using echo tracking methods are calibrated appropriately and accurately, the local PP achieved might be applied to more arterial sites and various subjects $[15,16]$.

Because distension waves can be obtained in subjects and at arterial sites where applanation tonometry and the transfer function are not reliable, or not possible for technical reasons, the assessment of the BP waveform via an ultrasonic examination of arteries in combination with an appropriate automatic image processing technique might provide a more practical alternative to applanation tonometry. The objective of the present study is to introduce and implement a novel noninvasive ultrasonic method to derive the pressure waveform of the carotid artery. The proposed technique is based on a theoretical equation relating the PP to the blood flow velocity and arterial wall distention, both of which can be obtained by processing consecutive sonograms. The resulting pressures are calibrated using the mean and diastolic BPs of the radial artery.

\section{Materials and Methods}

\section{Description of the Pressure Waveform Equation}

The association of a pressure change with the rapid velocity change across a pressure wave in pipe systems is well known. The basic physics of linear momentum yields the fundamental equation of a water hammer (Joukowsky's equation), to which accordingly, pressure and velocity changes are related as follows [17]:

$\Delta p=\rho(p w v) \Delta v$,

where $\rho$ denotes the fluid density, pwv represents the water hammer wave speed (pulse wave velocity), and $\Delta p$ and $\Delta v$ indicate the pressure and velocity changes relative to the corresponding initial values, respectively. Furthermore, the water hammer wave speed in an incompressible fluid within a flexible pipe is as follows:

$(p w v)^{2}=\frac{A \cdot \Delta p}{\rho \cdot \Delta \mathrm{A}}$,

where $\triangle A$ denotes the cross-sectional area (CSA) changes relative to the initial CSA.

Substituting $\mathrm{Eq}(2)$ in Eq (1) results in the following $[18,19]$ :

$\Delta p=\rho(\Delta v)^{2} \frac{A}{\Delta A}$.

In Eq (3), we assumed that irrespective of the velocity distribution throughout the CSA, all CSA elements undergo the same relative increase. Furthermore, the consideration that the fluid is frictionless [8] in a large vessel system is acceptable.

Ultrasonic duplex imaging provides arterial blood velocity changes during the cardiac cycle [20], while the arterial CSA can be achieved from sequences of B-mode ultrasonic images [21]. The uniqueness of the proposed method is to consider Eq (3) during cardiac cycles. By extracting the involved variables from ultrasonic image sequences 
of the carotid artery, PP $(\Delta p)$ might be achieved over the cardiac cycles.

To examine the clinical use of the proposed approach, consecutive sonograms of 20 subjects were analyzed using the following procedure.

\section{Subjects}

Participants in this study were 20 men (aged $36 \pm 9$ years) with no history of cardiovascular disease, hypertension, smoking, or diabetes. Participants were selected according to the clinical conditions presented [22]. All participants provided written informed consent for the examination, and the ethics committee of Tarbiat Modares University approved the procedure of this study.

\section{Data Acquisition}

After 10 minutes of recumbent resting in the supine position, the right common carotid artery of the subjects, $2 \mathrm{~cm}$ below the bifurcation of carotid artery, was scanned longitudinally with a Sonoline Antares digital ultrasound scanner (Siemens, Erlangen, Germany) equipped with a 5-13-MHz linear transducer with harmonic capability. The transducer was placed at the lateral side of the neck where the maximum lumen diameter appeared and the typical double lines of the artery could be seen. The Audio Video Interleave (AVI) format of an image sequence of the common carotid artery with a frame rate of $30 \mathrm{~Hz}$ was transferred to a PC for postprocessing. Each recording contained three cardiac cycles. A custom analysis program written in the MATLAB environment (Math Works, Natick, MA, USA) was used for extracting consecutive images in the Bitmap (BMP) format from AVI movies. The internal diameter was estimated offline by using the maximum gradient algorithm, designed in Ultrasound Laboratory of Tarbiat Modares University by MATLAB software. The mentioned algorithm allows a quantitative evaluation of the diameter changes by processing consecutive ultrasonic images. This algorithm was described in detail and validated elsewhere [23].

All images were processed by a custom MATAB program, in which the speckle noise was removed by setting an appropriate threshold for the grayscale level histogram $[18,24]$. Then, the envelope of the velocity profile was distinguished by the pixels after which the level value of at least 10 pixels was nonzero.

To extract the waveform of blood flow velocity, Doppler sonograms of the carotid arteries containing three cardiac cycles, from the same position expressed above, were recorded for all subjects. Recordings were made in the center of the artery, and the angle between the Doppler beam and the vessel orientation was chosen to be $60^{\circ}$ to measure the correct Doppler shifts [25]. All images were processed by a custom MATAB program, in which the speckle noise was removed according to the method described [24]. In brief, several small areas outside the Doppler spectrum were selected and their grayscale levels were evaluated. The lowest grayscale level was set as the threshold for the grayscale level histogram. Then, the envelope of the velocity profile was distinguished by the pixels after which the level value of at least 10 successive pixels was nonzero. This avoids the possibility of considering noise speckles to be a velocity profile component.

The internal diameter and blood flow velocity waveforms were recorded at different sample rates. Therefore, the recorded waveforms were decomposed into two parts: beginning to peak systolic and peak systolic to end diastolic. Then, the first portions of the waveforms were resampled at the sample rate of the maximum sample number existing in the first parts of the two waveforms. The second parts of the waveforms were resampled similarly. This ensures that the cardiac phases of the two waveforms are synchronized.

Arterial CSA was considered to be a circle and was calculated from the internal diameter. Arterial CSA, blood flow velocity, and CSA changes (instant waveforms minus the corresponding end diastolic values) during three cardiac cycles were substituted in Eq (3), and the PP waveform of the carotid artery was obtained during three cardiac cycles in all the subjects. The blood density was considered to be $1,060 \mathrm{~kg} / \mathrm{m}^{3}$ [26]. Because the diastolic blood pressure (DBP) is assumed to be constant throughout the large conduit arteries [3], the DBPs measured from the radial artery of the subjects by using a calibrated digital sphygmomanometer were added to the achieved PP waves in order to obtain the BP wave. The sphygmomanometer calibration was carried out using an SES (SES04 pump, Wild Heerbrugg, Heerbrugg, Switzerland) pump with the precision of $0.1 \mathrm{~mm} \mathrm{Hg}$ at three pressure values: 100, 200, and 280 $\mathrm{mm} \mathrm{Hg}$. The mean error of the digital sphygmomanometer for the three pressure values was $0.7 \%$. More details of the calibration are provided elsewhere [27].

The resulting waveforms were calibrated to the noninvasively measured mean radial artery pressure by using the following procedure in order to explore the potential of the proposed method to represent the noninvasively measured carotid pressure waveform.

\section{Calibration Method}

In general, pressure waveforms are calibrated by using the mean blood pressure (MBP) and DBP, assuming that they remain nearly constant throughout the arterial tree [3]. At the same time, the existence of a difference in the SBPs and PPs of the central and peripheral arteries should also be considered $[28,29]$.

In this study, the MBP of the subjects was estimated using the SBP and DBP measured from the radial arteries of the subjects by 
using a calibrated digital sphygmomanometer. In other words, the MBP was assumed to be a summation of the DBP and one-third of the PP [21].

To estimate the MBPs of the carotid arteries from the pressure curves obtained from image processing, the numeric integral of the processed curves was calculated. Then, we considered a linear correlation between the MBPs calculated from the radial artery and image processing. That is, Pearson's linear correlation between the MBP values resulting from two different methods could be written as follows:

$D B P+\frac{P P_{\text {rad }}}{3}=m\left(D B P+\frac{P P_{\text {pro }}}{3}\right)+b$,

where $m$ and $b$ denote the correlation line slope and the intercept of the correlation equation, respectively, and the subscripts rad and pro indicate the radial artery and image processing, respectively.

Moreover, according to the findings, the PP amplification between the central and the radial arteries is approximately $10-15 \mathrm{~mm} \mathrm{Hg}$ in either normotensive or hypertensive subjects $[8,27,28]$. Furthermore, an invasive measurement has shown that the radial systolic pressure is $12 \pm 1 \mathrm{~mm} \mathrm{Hg}$ higher than the central BP [30].

In this study, a mean value of $12 \mathrm{~mm} \mathrm{Hg}$ for the amplification between the PPs of the carotid and the radial arteries $\left(p p_{\text {rad }}=\right.$ $\left.p p_{a c t}+12\right)$ was considered. The calibrated factor ( $k$ ) was defined as a fraction of the actual and the processed PPs of the carotid artery $\left(k=\frac{P P_{\text {act }}}{P P_{\text {pro }}}\right)$

Therefore, two equations of the MBP correlation relation (Eq 4) and the PP amplification were substituted in the calibration factor definition to extract the subject-specific calibration factor.

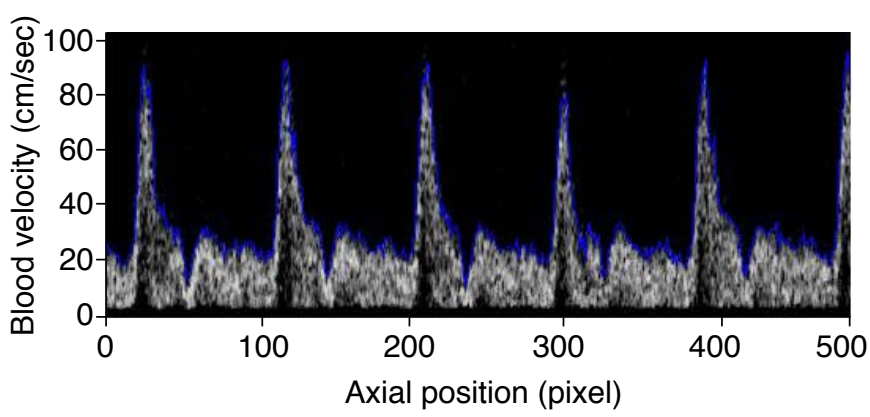

A

Fig. 1. Envelope and velocity profiles of the blood flow.

In the envelope profile of the blood flow velocity (A) and the velocity profile extracted from Doppler ultrasonic image processing (B), the automatic tracing procedure extracted the real velocity waveform and the resulted curve fitted the actual profile.

e-ultrasonography.org

Ultrasonography 36(2), April 2017 
To employ the previously described noninvasive calibration method, Pearson's linear correlation between MBPs of the waveforms obtained by image processing and those measured on the basis of radial artery pressures was calculated. The correlation between the MBPs of image processing-based pressure curves and those measured from radial artery pressures is depicted in Fig. 3. According to the resultant linear correlation equation, the $m$ and

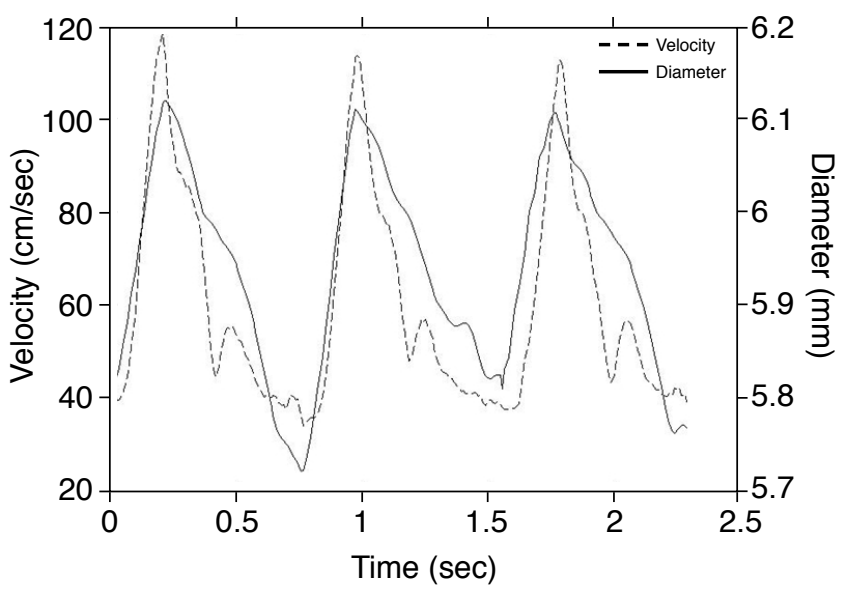

Fig. 2. Synchronized internal diameter waveform (solid line) and the profile of blood flow velocity (dash line) of the right common carotid artery of a subject. The internal diameter waveform was obtained from consecutive sonograms processed by the maximum gradient algorithm, while the profile of the blood flow was obtained from Doppler sonogram.

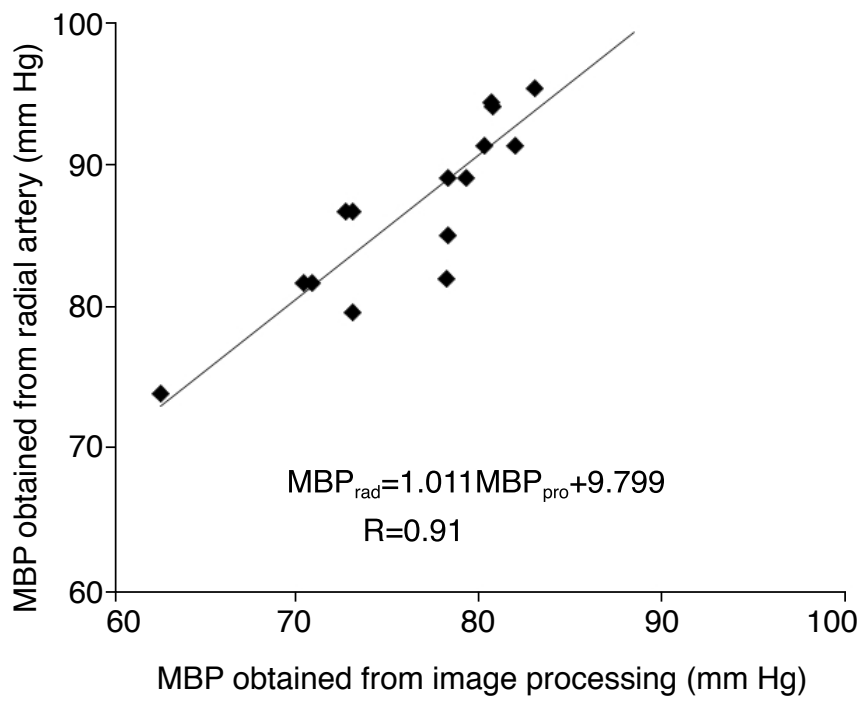

Fig. 3. Correlation between mean blood pressures (MBPs) of image processing $\left(\mathrm{MBP}_{\text {pro }}\right)$ based pressure waveforms and those measured from radial artery $\left(\mathrm{MBP}_{\text {rad }}\right)$. Values of the slop $(m)$ and intercept $(b)$ are obtained from the correlation results and are used to derive the calibration factor of each subject. $b$ values used for the calibration factor calculation were 1.011 and 9.799, respectively (Fig. 3).

To achieve the calibrated PP wave, the calibration factor of each subject was multiplied with his own uncalibrated PP waveform of the common carotid artery obtained from sonograms (Fig. 4).

To assess the agreement between the calibrated PPs and the corresponding values measured via a standard sphygmomanometer, a scatterplot of the Bland-Altman analysis was drawn and the differences between the PPs measured by the abovementioned methods were plotted against the average values of the differently achieved PPs (Fig. 5). According to the scatterplot, the PPs obtained from image processing and those calibrated using the calibration factor have a good coherence with the PPs measured using a standard sphygmomanometer at the radial artery.

The mean absolute difference of the calibrated PPs and the clinically measured PPs was $-1.333 \pm 6.548 \mathrm{~mm} \mathrm{Hg}$, and the mean value of the calibration factors was $2.224 \pm 0.721$. The resulting differences fell within the Association for the Advancement of Medical Instrumentation (AAMI) recommended limits of acceptance between the standard and the automated methods of pressure measurement [32].

\section{Discussion}

Accurate computerized delineation of a pressure wave seems to be a novel idea for daily cardiovascular health monitoring $[33,34]$.

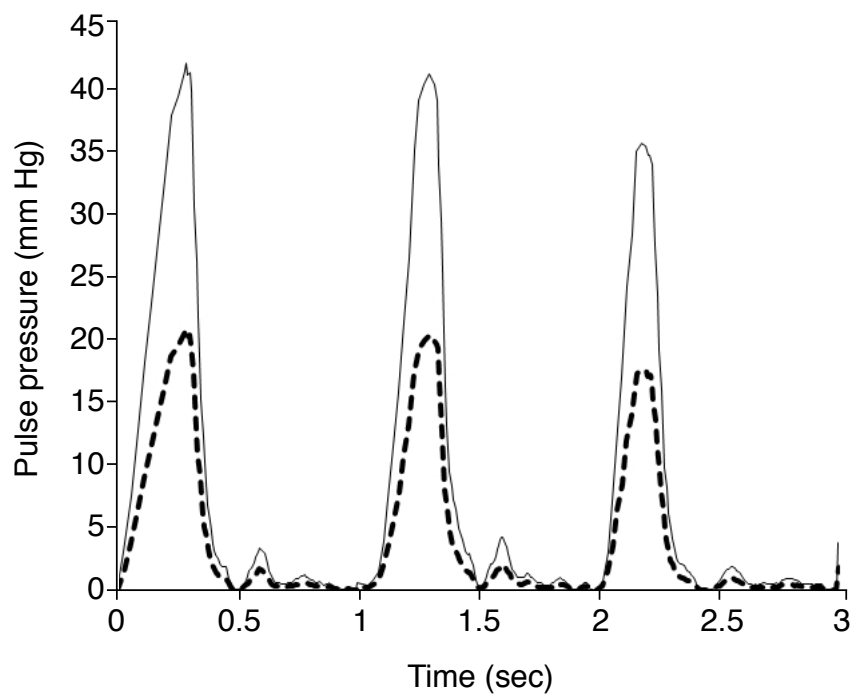

Fig. 4. Pulse pressure waveforms of the common carotid artery of a healthy subject. In accordance with the calibration factor definition, multiplying the uncalibrated pulse pressure (PP) waveform (dash line) of each subject by his own calibration factor leaded to the calibrated PP wave (solid line). 


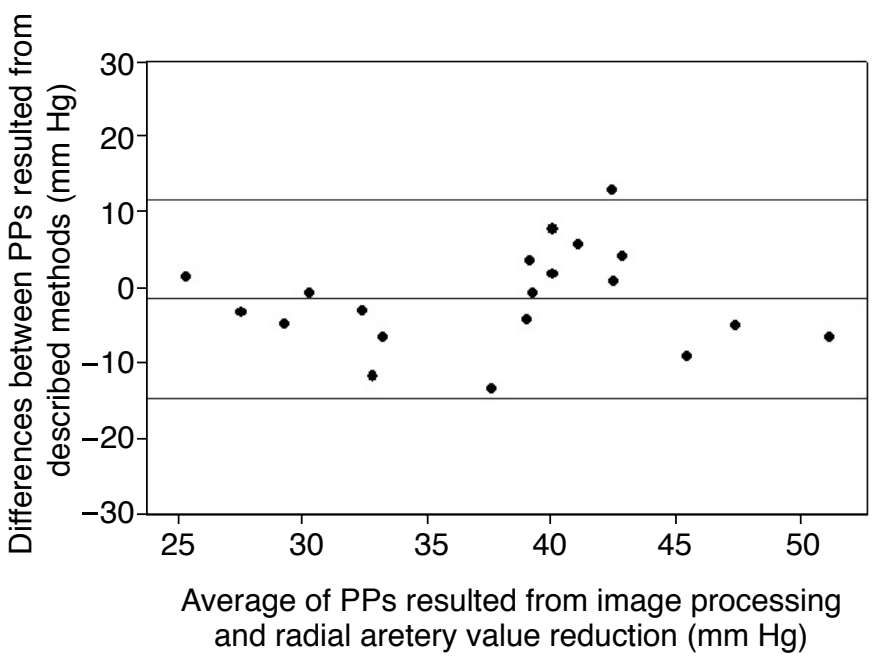

Fig. 5. Bland-Altman scatter plot of the difference between pulse pressures (PPs) estimated based on ultrasound image processing method and those obtained from radial artery PPs. The middle line is the mean difference between the results of two methods, the outer lines correspond to 2SD around the mean difference. SD stands for standard deviation of the differences between the calibrated PPs obtained from image processing and those obtained from radial artery.

Today, ultrasonic inspection is widely accepted as a noninvasive technique for general clinical applications. The ultrasound imaging feasibility of most vascular sites, cost effectiveness and its incorporation into cardiovascular imaging examinations without any additional equipment [10] encourage the idea of using the same imaging test to obtain an arterial pressure waveform, which might promote anatomical and functional information about arterial health. In this study, we established a novel ultrasonic method for pressure waveform achievement. Thus far, the results of the attempts related to the development of noninvasive ultrasonic techniques for pressure wave measurement have been notable.

Olesen et al. [35] applied an ultrasonic method for estimating 2D hemodynamic pressure gradients in a flow model, which mimicked the carotid bifurcation having $70 \%$ occlusion at the beginning of the internal carotid artery. Pressure gradients were calculated using Navier-Stokes equations in which the vector velocity fields were obtained by processing ultrasound radiofrequency data. The results were compared to the pressure resultant from 3D finite element modeling of a phantom. In the mentioned study, steady flow conditions were set up and the gravitational force and the out-ofplane velocity component were neglected [35].

Haluska et al. [10] acquired the pressure wave of the carotid artery from TDI and compared it with the tonometry results. They showed that there is a high correlation between the results of TDI- based pressure waveforms and those of tonometry with a difference of $5.36 \pm 5.5 \mathrm{~mm} \mathrm{Hg}$ in systolic pressure values [10].

Moreover, studies in which the BP waveforms were assessed from echo tracking distension waves calibrated linearly or exponentially, reported that there is an underestimation of at least $2.5 \pm 5 \mathrm{~mm} \mathrm{Hg}$ in the carotid artery PP [15].

Consistently, the MBP derived from our novel method correlated well with the radial artery MBP $(R=0.91)$ and the mean absolute difference of the calibrated PPs and the clinically measured PPs was $-1.333 \pm 6.548 \mathrm{~mm} \mathrm{Hg}$, which seems to fall within the reported error regions.

All noninvasively acquired arterial pressure waveforms require calibration to a known BP, which may impose a potential source of measurement errors, particularly when inaccurate pressures are used for noninvasive calibration. However, noninvasive calibration is widely used in the literature and a literature review showed that central PP errors vary in the range of -32.5 to $8.1 \mathrm{~mm} \mathrm{Hg}$ when noninvasive calibration to peripheral BP is applied [3]. Furthermore, Agnoletti et al. [36] showed that pressure waves of different arteries obtained as a result of applanation tonometry, which were calibrated using four different noninvasive methods, lead to a difference of $14-18 \mathrm{~mm} \mathrm{Hg}$ in PPs.

Hope et al. [37] measured the noninvasive radial pressure waveforms and compared the impact of invasive and noninvasive calibration methods on the same central pressure waveform derived from a transfer function. They showed that noninvasive calibration resulted in an underestimation of $7 \pm 12 \mathrm{~mm} \mathrm{Hg}$ in the estimation of the central systolic pressure. They found a significant difference between the radial waveform calibrated invasively and noninvasively and suggested that the differences were due to the invasive and noninvasive diastolic pressure of the aorta [37].

Van Bortel et al. [9] reported that noninvasively calibrated arterial distension waves caused by echo tracking are $3.4 \pm 6.9 \mathrm{~mm} \mathrm{Hg}$ lower than the PP waves obtained from invasively calibrated tonometry. However, the differences between invasively and noninvasively derived and calibrated pressure waveforms are inevitable. Any difference between our image processing-derived pressure waveforms and the invasively derived ones may be attributed to the assumption that both the mean and the diastolic pressures remain unchanged throughout the arterial tree. However, the notion of exactly the same mean and diastolic pressure values from the central to the peripheral artery holds true only in invasive measurements of the central and peripheral BP [3].

Further, although a mathematical manipulation of the radial artery pressure waveform predicts the peak and through pressure well, the exact shape achievement requires an invasive measurement. Moreover, errors caused by the mathematical modeling between the 
peripheral and the central pressure waveform might be challenging $[3,6]$. Furthermore, the transfer function method is sensitive to noninvasive calibration errors and the accuracy of the results is affected by the calibration errors [36].

For technical reasons, applanation tonometry and the transfer function are not reliable, or not possible at all arterial sites, and the method proposed in this study, assessing the BP waveform via an ultrasonic examination of the arteries by using an appropriate automatic image processing technique, can provide a more practical alternative to applanation tonometry.

The proposed method as a noninvasive BP measurement technique suffers from the same limitation of calibration errors. In the present study, invasively measured BPs were not available and this may pose errors. Further, the error of the acquired pressures in our study may be associated partly with the incorrect estimation of the centerline velocity profile caused by the insonation angle error [24] and the weakness of the $33 \%$ rule as compared to the precise method of measuring MBP via an intra-arterial catheter [35].

Beulen et al. [38] extracted the local pressure waveforms in a phantom set up. Their method was based on a particle image velocimetry technique applied to the high-frame-rate ultrasound radio frequency data. This way, they circumvented the Doppler flow velocity errors because their implemented method required no beam steering and the relation between the pulse wave velocity (PWV) and the local pressure was derived with the assumption of a linear elastic thin-walled vessel. In their study, PWV was extracted by a flow-area (Q-A) method, which is restricted to the reflection-free periods of the cardiac cycle. It was shown that for carotid-like pulses, the reflection-free part of the Q-A loop was not clear and highsample-rate techniques were required to obtain adequate sample points over the linear section of the loop [38].

A further potential limitation is the use of the mean value of 12 $\mathrm{mm} \mathrm{Hg}$ for PP amplification. PP amplification has been reported to fall with aging [39]. Considering different values for younger and older subjects may improve the accuracy of the obtained pressure.

It is worth noting that according to AAMI recommendations, the acceptance upper limit between the difference of the standard and the automated methods for BP measurement is \pm 5 and $\pm 8 \mathrm{~mm} \mathrm{Hg}$ for mean and SD, respectively [31].

Another limitation from which the present study suffers is that this study consisted of only normal carotid arteries without pathological changes, such as the degree of carotid stenosis and the arterial CSA was assumed to be a circle, while this assumption has been a large trial in regions having irregular and asymmetric stenotic plaques. Nevertheless, in these regions, it might be required to record additional consecutive ultrasonic images from the transverse view of the artery, and then, arterial CSA can be outlined using segmentation methods. However, the same procedure can be used for the subjects who have hypo/hypertension; that is, once the correlation equation (slope, intercept) between the MBP values obtained from image processing and values measured from the radial artery was calculated for a group of subjects suffering from hypo/hypertension, Eq (5) is applicable to extract the patient-specific calibration factor for any typical patient having the same disease.

In the present study, the blood is assumed to be a perfect fluid. Therefore, the proposed method is expected to provide an appropriate inter-subject comparison of the pressure waveform shape and characteristics.

In spite of all the limitations, the error in the PPs obtained from our ultrasonic technique is similar to the reported results of other publications using different methods.

It has been suggested that when a pressure wave is extracted from arterial wall displacement, no phase delay due to spatial separation or filter characteristics will affect the result [40]. Nonetheless, the methods in which the pressure curve is derived purely from a diameter wave neglect the effect of the blood flow and the viscoelastic properties of arteries on the pressure waveform and generally assign an exponential relationship between the diameter and the pressure [39]. However, theoretically, both the blood flow and wall properties determine the pressure waveform characteristics [18]. To the best of our knowledge, this is the first time that a pressure waveform is extracted practically from a theoretical equation considering both the blood flow and wall effects. This technique can be implemented for all arterial sites. Extraction of the other sites pressure waveform, particularly that of the radial artery, via the proposed technique in the present study and a comparison of the results with those of the carotid artery are suggested for future studies.

In conclusion, the results of this study suggest that arterial PP waveforms can be assessed using a noninvasive ultrasonic examination of arteries, promoting a noninvasive technique for clinical applications. We believe that the proposed method, having a high agreement with the literature, would overcome the drawback of simultaneously assessing pressure and diameter waves at specific sites for the estimation of the total arterial compliance and it would seem to overcome the reproducibility problems of common carotid artery tonometry with technical and anatomical causes.

ORCID: Effat Soleimani: http://orcid.org/0000-0003-3376-7323; Manijhe MokhtariDizaji: http://orcid.org/0000-0003-1598-2038

\section{Conflict of Interest}

No potential conflict of interest relevant to this article was reported. 


\section{Acknowledgments}

This study was approved by Faculty of Medical Sciences of Tarbiat Modares University.

\section{References}

1. Waddell TK, Dart AM, Medley TL, Cameron JD, Kingwell BA. Carotid pressure is a better predictor of coronary artery disease severity than brachial pressure. Hypertension 2001;38:927-931.

2. McEniery CM, Cockcroft JR, Roman MJ, Franklin SS, Wilkinson IB. Central blood pressure: current evidence and clinical importance. Eur Heart J 2014;35:1719-1725.

3. Cheng HM, Lang D, Tufanaru C, Pearson A. Measurement accuracy of non-invasively obtained central blood pressure by applanation tonometry: a systematic review and meta-analysis. Int I Cardiol 2013;167:1867-1876.

4. Nelson MR, Stepanek J, Cevette M, Covalciuc M, Hurst RT, Tajik AJ. Noninvasive measurement of central vascular pressures with arterial tonometry: clinical revival of the pulse pressure waveform? Mayo Clin Proc 2010;85:460-472.

5. Fetics $B$, Nevo $E$, Chen $C H$, Kass DA. Parametric model derivation of transfer function for noninvasive estimation of aortic pressure by radial tonometry. IEEE Trans Biomed Eng 1999;46:698-706.

6. Chen $\mathrm{CH}$, Nevo E, Fetics B, Pak PH, Yin FC, Maughan WL, et al. Estimation of central aortic pressure waveform by mathematical transformation of radial tonometry pressure: validation of generalized transfer function. Circulation 1997;95:1827-1836.

7. Segers $P$, Carlier S, Pasquet A, Rabben SI, Hellevik LR, Remme E, et al. Individualizing the aorto-radial pressure transfer function: feasibility of a model-based approach. Am J Physiol Heart Circ Physiol 2000;279:H542-H549.

8. Nichols WW, O'Rourke MF, Vlachopoulos C. McDonald's blood flow in arteries: theoretical, experimental and clinical principles. 6th ed. London: Hodder Arnold, 2011.

9. Van Bortel LM, Balkestein EJ, van der Heijden-Spek JJ, Vanmolkot FH, Staessen JA, Kragten JA, et al. Non-invasive assessment of local arterial pulse pressure: comparison of applanation tonometry and echo-tracking. J Hypertens 2001;19:1037-1044.

10. Haluska BA, Jeffriess L, Mottram PM, Carlier SG, Marwick TH. A new technique for assessing arterial pressure wave forms and central pressure with tissue Doppler. Cardiovasc Ultrasound 2007;5:6.

11. Naik V, Gamad RS, Bansod PP. Carotid artery segmentation in ultrasound images and measurement of intima-media thickness. Biomed Res Int 2013;2013:801962.

12. Zhang YH, Gao Y, Su BL. Assessment of carotid arterial wall elasticity in type 2 diabetes mellitus patients with microalbuminuria by realtime ultrasound elastography. Int J Endocrinol 2012;2012:340974.

13. Tortoli P, Bettarini R, Guidi F, Andreuccetti F, Righi D. A simplified approach for real-time detection of arterial wall velocity and distension. IEEE Trans Ultrason Ferroelectr Freq Control 2001;48:1005-1012.

14. Golemati S, Sassano A, Lever MJ, Bharath AA, Dhanjil S, Nicolaides AN. Carotid artery wall motion estimated from B-mode ultrasound using region tracking and block matching. Ultrasound Med Biol 2003;29:387-399.

15. Graf S, Craiem D, Armentano RL. Non invasive assessment of carotid and femoral arterial pressure using B-mode ultrasound diameter waveforms. Conf Proc IEEE Eng Med Biol Soc 2012;2012:56105613.

16. Vermeersch SJ, Rietzschel ER, De Buyzere ML, De Bacquer $D$, De Backer G, Van Bortel LM, et al. Determining carotid artery pressure from scaled diameter waveforms: comparison and validation of calibration techniques in 2026 subjects. Physiol Meas 2008;29:1267-1280.

17. Mays LW. Hydraulic design handbook. New York: McGraw-Hill, 2000.

18. Ghidaoui MS, Zhao M, McInnis DA, Axworthy DH. A review of water hammer: theory and practice. Appl Mech Rev 2005;58:49-76.

19. Laffon $E$, Marthan $R$, Montaudon $M$, Latrabe $V$, Laurent $F$, Ducassou D. Feasibility of aortic pulse pressure and pressure wave velocity MRI measurement in young adults. J Magn Reson Imaging 2005;21:53-58.

20. Zhang DY, Zuo WM, Zhang D, Zhang HZ, Li NM. Wrist blood flow signal-based computerized pulse diagnosis using spatial and spectrum features. J Biomed Sci Eng 2010;3:361-366.

21. Bussy C, Boutouyrie P, Lacolley P, Challande P, Laurent S. Intrinsic stiffness of the carotid arterial wall material in essential hypertensives. Hypertension 2000;35:1049-1054.

22. Schmidt-Trucksass A, Grathwohl D, Schmid A, Boragk R, Upmeier C, Keul J, et al. Assessment of carotid wall motion and stiffness with tissue Doppler imaging. Ultrasound Med Biol 1998;24:639-646.

23. Soleimani E, Dizaji MM, Saberi H. Carotid artery wall motion estimation from consecutive ultrasonic images: comparison between Block-Matching and Maximum-Gradient algorithms. J Tehran Heart Cent 2011;6:72-78.

24. Tschirren J, Lauer RM, Sonka M. Automated analysis of Doppler ultrasound velocity flow diagrams. IEEE Trans Med Imaging 2001;20:1422-1425.

25. Thijssen DH, Black MA, Pyke KE, Padilla J, Atkinson G, Harris RA, et al. Assessment of flow-mediated dilation in humans: a methodological and physiological guideline. Am J Physiol Heart Circ Physiol 2011;300:H2-H12.

26. Kock SA, Nygaard JV, Eldrup N, Frund ET, Klaerke A, Paaske WP, et al. Mechanical stresses in carotid plaques using MRI-based fluidstructure interaction models. J Biomech 2008;41:1651-1658.

27. Moladoust H. Evaluation of physical and mechanical properties of the myocardium muscle in normal and patients with coronary stenosis using echocardiographic images. PhD dissertation. Tehran: 
Tarbiat Modares University, 2009.

28. Safar ME, Kakou A. Carotid and brachial blood pressure: measurements in hypertensive subjects. Rev Bras Hipertens 2008;15:122-124.

29. Benetos A, Thomas F, Joly L, Blacher J, Pannier B, Labat $C$, et al. Pulse pressure amplification a mechanical biomarker of cardiovascular risk. J Am Coll Cardiol 2010;55:1032-1037.

30. Pauca AL, Wallenhaupt SL, Kon ND, Tucker WY. Does radial artery pressure accurately reflect aortic pressure? Chest 1992;102:11931198.

31. Bland JM, Altman DG. Statistical methods for assessing agreement between two methods of clinical measurement. Lancet 1986;1:307310.

32. White WB, Berson AS, Robbins C, Jamieson MJ, Prisant LM, Roccella $\mathrm{E}$, et al. National standard for measurement of resting and ambulatory blood pressures with automated sphygmomanometers. Hypertension 1993;21:504-509.

33. Li BN, Dong MC, Vai MI. On an automatic delineator for arterial blood pressure waveforms. Biomed Signal Process Control 2010;5:76-81.

34. Al-Jumaily AM, Lan $\mathrm{H}$, Stergiopulos N. Brachial artery waveforms for automatic blood pressure measurement. J Biomech 2013;46:506-
510.

35. Olesen J, Traberg M, Pihl M, Jensen J. Noninvasive estimation of 2-D pressure gradients in steady flow using ultrasound. IEEE Trans Ultrason Ferroelectr Freq Control 2014;61:1409-1418.

36. Agnoletti D, Zhang Y, Salvi P, Borghi C, Topouchian J, Safar ME, et al. Pulse pressure amplification, pressure waveform calibration and clinical applications. Atherosclerosis 2012;224:108-112.

37. Hope SA, Meredith IT, Cameron JD. Effect of non-invasive calibration of radial waveforms on error in transfer-function-derived central aortic waveform characteristics. Clin Sci (Lond) 2004;107:205-211.

38. Beulen BW, Bijnens N, Koutsouridis GG, Brands PJ, Rutten MC, van de Vosse FN. Toward noninvasive blood pressure assessment in arteries by using ultrasound. Ultrasound Med Biol 2011;37:788797.

39. Avolio AP, Van Bortel LM, Boutouyrie P, Cockcroft JR, McEniery CM, Protogerou $A D$, et al. Role of pulse pressure amplification in arterial hypertension: experts' opinion and review of the data. Hypertension 2009;54:375-383.

40. Meinders JM, Hoeks AP. Simultaneous assessment of diameter and pressure waveforms in the carotid artery. Ultrasound Med Biol 2004;30:147-154. 